\section{$\underset{\substack{\text { hommes } \\ \text { \& migrations }}}{ }$}

\section{Hommes \& migrations}

Revue française de référence sur les dynamiques

migratoires

$1283 \mid 2010$

Cuisines et dépendances

\title{
Alimentation et migration : une relation révélatrice
}

\section{Jacques Barou}

\section{(2) OpenEdition \\ Journals}

\section{Édition électronique}

URL : http://journals.openedition.org/hommesmigrations/980

DOI : 10.4000/hommesmigrations.980

ISSN : 2262-3353

\section{Éditeur}

Musée national de l'histoire de l'immigration

\section{Édition imprimée}

Date de publication : 1 janvier 2010

Pagination : 6-11

ISBN : 978-2-919040-04-9

ISSN : $1142-852 X$

\section{Référence électronique}

Jacques Barou, «Alimentation et migration : une relation révélatrice », Hommes \& migrations [En ligne], 1283 | 2010, mis en ligne le 29 mai 2013, consulté le 10 décembre 2020. URL : http://

journals.openedition.org/hommesmigrations/980; DOI : https://doi.org/10.4000/hommesmigrations. 980 


\section{Alimentation et migration : une relation révélatrice}

Par Jacques Barou,
chercheur, docteur en anthropologie, chargé de recherche au CNRS,

UMR PACTE, Grenoble

L'alimentation fait depuis quelques années l'objet de nombreuses recherches impliquant les sciences humaines, indépendamment ou parallèlement aux sciences de la vie et de la terre. Ces recherches sont parfois guidées par des questions économiques majeures et des questions de société. À l'heure où des pénuries alimentaires se font sentir dans le monde, où la vie moderne incite au développement d'une certaine malbouffe et d'un appauvrissement des goûts, la question de l'alimentation n'est en rien une question banale. Il s'agit au contraire d'un domaine potentiellement riche de découvertes susceptibles d'éclairer divers aspects de la vie des sociétés contemporaines : les dynamiques identitaires, les relations commerciales, les modes de convivialité, les transformations des rapports sociaux et familiaux... C'est à cette exploration des diverses facettes de la question alimentaire que ce numéro entend contribuer à partir de textes qui dévoilent les divers liens qu'entretiennent l'alimentation et les migrations. Curieusement peu étudiée, cette thématique s'avère porteuse de nombreux enseignements quant au devenir des différentes migrations et aux influences qu'elles exercent et reçoivent de la part de leurs sociétés de départ et de leurs sociétés d'accueil.

\section{Alimentation et ressources économiques et sociales}

Pour l'immigré, les métiers de l'alimentation constituent souvent une première ressource pour assurer sa survie dans le lieu où il vient d'arriver. Des activités comme la distribution de denrées de base offrent un accès rapide à un travail rémunéré. En témoigne l'importance de petits métiers comme ceux de porteur d'eau ou de laitier exercés par les immigrés auvergnats dans le Paris du XIX siècle ou par d'autres immigrés "de l'intérieur" dans les grandes villes de l'Asie et de l'Afrique d'aujourd'hui. 
Ces activités peuvent être à l'origine d'une réussite sociale dans les divers métiers du commerce alimentaire, de la restauration ou des débits de boissons quand les migrants savent conjuguer ressources alimentaires, ressources sociales et ressources identitaires.

De la même manière, Cyprien Coffi Aholou montre comment les migrants kabiyé, losso ou moba, originaires du nord du Togo, se construisent une trajectoire sociale ascendante dans la capitale Lomé, à partir de la vente d'une bière artisanale, le tchoukoutou, spécialité de leur région d'origine. En diffusant cette boisson dans des cabarets ouverts à bon compte dans des quartiers populaires, ils renforcent à la fois leur lien identitaire et offrent un lieu de convivialité apprécié par l'ensemble des citadins.

\section{Alimentation, adaptation et mémoire}

Le renforcement de l'identité des migrants autour des pratiques alimentaires est abordé à divers stades par plusieurs contributeurs. Mohamadou Sall observe que les familles sénégalaises originaires du Fouta Toro, immigrées à Mantes-la-Jolie, se sont organisées pour perpétuer la consommation de plats typiques de leur région d'origine. Leur pratique alimentaire apparaît ainsi comme immuable, répondant à une nécessité de résister au choc de la transplantation, même après plusieurs années de vie en France. La mémoire du pays est ainsi cultivée à table et le lien identitaire se renforce par la réalisation et la consommation de recettes caractéristiques d'une région rurale au profil très éloigné du lieu d'immigration.

Pour des populations nées en France, la consommation des plats du pays d'origine n'empêche pas l'intérêt pour des produits caractéristiques de l'environnement alimentaire actuel. C'est ce qu'analyse Hatice Soyturk à propos des pratiques alimentaires métissées des adolescents d'origine turque vivant en Alsace. Ils exaltent les vieilles recettes familiales qu'ils souhaitent pouvoir continuer à consommer dans leur composition authentique, tout en sacrifiant largement aux modes de consommation propres à leur génération. Clients assidus des fast-foods, où ils participent au mode de consommation propre à leur classe d'âge, ils n'en demeurent pas moins attachés aux plats traditionnels qui les relient à leurs origines.

L'adaptation à un nouveau contexte alimentaire constitue aussi l'objet de l'article de Frédérique Giraud qui observe comment les étudiants étrangers de Lyon organisent leurs pratiques alimentaires pendant leur séjour en France, en repérant dans les commerces locaux les substituts acceptables aux produits qu'ils ne pourront pas facilement ramener de chez eux. 


\section{Migrations et patrimoine gastronomique}

Mais les nourritures et les recettes importées par les immigrés ne sont pas toujours destinées à rester dans le seul cercle des communautés qui les ont amenées avec elles. Progressivement, elles se diffusent au-dehors de ces premiers cercles de consommateurs et elles se transforment au contact d'autres influences. C'est ce que fait ressortir Raoul Matta à propos de la constitution du patrimoine gastronomique péruvien, reconnu aujourd'hui comme l'un des plus riches et des plus prestigieux d'Amérique latine. Aux produits et aux recettes autochtones se sont mélangées avec bonheur des influences introduites par les diverses vagues migratoires qu'a connues le pays. À une cuisine criolla, résultant de la rencontre entre les produits amérindiens et les recettes appréciées des conquérants espagnols, sont venues s'ajouter au $\mathrm{XIX}^{\mathrm{e}}$ et $\mathrm{XX}^{\mathrm{e}}$ siècle des influences chinoises, italiennes et japonaises, aboutissant en fin de compte à une cuisine apte plus que d'autres à devenir internationale, sans rien perdre se son raffinement ni de sa créativité.

L'intérêt universel que peut présenter un patrimoine gastronomique particulier se retrouve dans l'article de Carole Lemee. Pour les juifs d'Europe orientale qui ont survécu au génocide, retrouver le patrimoine culinaire yiddish permet certes de renforcer l'identité communautaire par une reconnexion symbolique avec les ancêtres disparus. Mais cette redécouverte favorise aussi la prise de conscience des proximités qu'un tel patrimoine présente avec les cuisines des régions dans lesquelles il s'est constitué. Le retour à la consommation de cette cuisine-là n'aboutit pas à une forme de repli religieux, mais à la communion avec un univers beaucoup plus large de juifs de pratiques et de niveaux de conviction différents, ainsi qu'avec des populations non juives d'Europe orientale qui, elles-mêmes, retrouvent dans la cuisine yiddish des éléments propres à leurs traditions gastronomiques.

\section{Entre sacré et profane}

La fonction de retour à l'identité religieuse que peut présenter l'alimentation est bien mise en valeur dans l'article de Michel Karine. En l'occurrence, il s'agit moins d'un retour que d'un nouveau départ. Au sein des familles de juifs russophones immigrées en Allemagne, ce sont les jeunes qui redécouvrent les préceptes religieux en matière d'alimentation. Alors que leurs parents, habitués à une consommation indifférente aux interdits et recommandations judaïques au cours de leur vie en URSS, maintiennent des pratiques alimentaires profanes, ces jeunes ont à cceur d'opérer une nouvelle fondation identitaire dans un nouveau pays d'accueil en 
respectant de manière stricte les obligations de la kashrout. Ils parviennent même à imposer leurs pratiques alimentaires à leurs parents, réalisant ainsi une inversion de transmission, ce qui est caractéristique d'une volonté de construire une nouvelle base communautaire oublieuse du passé soviétique et désireuse de se démarquer de l'ensemble de la société d'immigration.

Maria Inacia d'Avila, Régina Andrade et Claudia Cavas explorent un cheminement inverse à propos de la cuisine pratiquée autour du culte du candomblé au Brésil. Initialement, il s'agit de recettes correspondant aux goûts des divers dieux des panthéons africains importés par les esclaves amenés au Brésil depuis le golfe du Bénin. Ces plats étaient offerts aux dieux dans le cadre de cérémonies rassemblant les adeptes sous la conduite des "mères de saints". Plusieurs de ces femmes prêtresses ou disciples des orixas ont été par la suite employées comme cuisinières dans des familles bourgeoises. Elles ont introduit dans les cuisines familiales les recettes inspirées des cultes afro-brésiliens, assurant ainsi le passage du sacré au profane, du communautaire au national.

\section{Permanence et changement}

L'évolution des pratiques alimentaires des migrants est aussi un reflet de la manière dont les migrants vivent les changements qui agitent la société au sein de laquelle ils se trouvent. Certains, pour résister à ces changements, s'accrochent à une habitude culinaire qui leur semble rassurante. L'usage de la Cocotte-minute par les ménagères immigrées du Maghreb, que décrit Aurélie Brayet, constitue une pratique bien intégrée au mode de préparation des plats traditionnels. L'utilisation d'un tel ustensile présente l'avantage à la fois d'une continuité, avec des habitudes de plats très mijotés ramenées du pays d'origine, et d'une bonne adaptation à la vie moderne, grâce à la réduction du temps de préparation et au bruit dégagé par l'appareil qui avertit la cuisinière de l'état du plat et la rassure par son sifflement régulier. Cette forte appropriation affective de l'objet leur permet de passer d'un milieu à un autre, d'un rythme à un autre, sans être trop perturbées par le changement.

Le texte d'Olga Bronnikova nous dévoile deux niveaux de vécu du changement alimentaire chez les Russes des classes moyennes immigrées dans ces villes globales que sont Paris et Londres. Ceux qui n'ont connu que l'URSS et sa cuisine industrielle standardisée ont du mal à s'adapter à un environnement alimentaire extrêmement diversifié, avec une présence de nourritures exotiques provenant de toutes les parties du monde. Ceux qui ont connu la Russie postsoviétique sont plus habitués à la diversité de l'offre alimentaire et plus intéressés par la découverte de nouveaux 
goûts. C'est aussi dans ces villes globales que ces deux catégories peuvent découvrir la cuisine russe traditionnelle oubliée pendant la période soviétique. Le changement prend ainsi l'aspect d'un retour.

\section{Alimentation et modernité}

Mais la ville moderne, de par les rythmes qu'elle impose et les offres en produits alimentaires industriels qu'elle propose, peut aussi occasionner la perte de certaines pratiques alimentaires que les immigrés ont ramenées de chez eux. Certains sont parfois amenés à oublier ces pratiques au profit d'une consommation de plats stéréotypés susceptibles de créer des problèmes sanitaires. Point n'est besoin pour cela de changer de pays. Le passage de la province à la grande ville peut être un facteur de déculturation alimentaire. C'est ce phénomène qu'analysent à Yaoundé JeanMarie Essomba, Mbonji Edjenguèlè, Patrick Pasquet et Annie Hubert à propos des migrants béti et bamiléké. Les pratiques alimentaires des migrants leur paraissent de moins en moins héritées mais de plus en plus métissées sous diverses influences dont celle, dominante, de la consommation de plats surgelés et importés d'Europe. Les changements dans les pratiques alimentaires sont appréhendés par Chantal Crenn et Isabelle Téchoueyres, dans une perspective d'anthropologie dynamique, à propos des élites malgaches immigrées en France. Les auteurs observent plusieurs vaet-vient entre des pratiques d'ici et de là-bas, des nostalgies du passé et des influences du présent. Pour ces catégories socialement privilégiées, le fait de "manger à la française" est un héritage de la génération précédente qui, à l'époque coloniale, avait adopté les habitudes culinaires du groupe dominant, pour en faire un signe de bonne éducation et de raffinement, propres à renforcer leur image élitaire. Le séjour en France est l'occasion de redécouvrir la cuisine malgache, sans pour autant la pratiquer autrement que par la consommation de quelques ingrédients de base. Cela suffit toutefois pour la mythifier et en faire une alimentation "naturelle". Mais un retour au pays fait naitre un regard beaucoup plus critique sur cette cuisine qui ne correspond pas aux préoccupations diététiques que les mangeurs ont acquis dans la France d'aujourd'hui.

En rendant compte de ces va-et-vient identitaires exprimés par les changements dans les pratiques alimentaires, en montrant aussi comment les migrations sont une occasion de rencontre culinaire et de métissage gastronomique et comment les activités liées à l'alimentation se retrouvent en divers lieux et temps comme opportunités de travail et d'ascension sociale, ce numéro apporte un éclairage sur plusieurs aspects de la relation entre alimentation et migration. 
